\title{
Statistical Inference of Truncated Weibull-Rayleigh Distribution: Its Properties and Applications
}

\author{
Eman H. Khalifa, Dina A. Ramadan, B. S. El-Desouky \\ Department of Mathematics, Faculty of Science, Mansoura University, Mansoura, Egypt \\ Email: emooookhalifa123@gmail.com,dina_ahmed2188@yahoo.com,b_desouky@yahoo.com
}

How to cite this paper: Khalifa, E.H., Ramadan, D.A. and El-Desouky, B.S. (2021) Statistical Inference of Truncated Weibull-Rayleigh Distribution: Its Properties and Applications. Open Journal of Modelling and Simulation, 9, 281-298. https://doi.org/10.4236/ojmsi.2021.93019

Received: March 25, 2021

Accepted: July 17, 2021

Published: July 20, 2021

Copyright (c) 2021 by author(s) and Scientific Research Publishing Inc. This work is licensed under the Creative Commons Attribution International License (CC BY 4.0). http://creativecommons.org/licenses/by/4.0/

\begin{abstract}
In this paper, we derived a new distribution named as truncated Weibull Rayleigh (TW-R) distribution. Its characterization and statistical properties are obtained, such as reliability function, hazard function, reversed hazard rate function, cumulative hazard rate function, quantile function, rth moment, incomplete moments, Rényi and q entropies and order statistic. Parameter estimation is implemented using method of maximum-likelihood estimation and Fisher information matrix is derived. Finally, application of the presented new distribution to a real data representing the failure times of 63 airbcraft Windshield is given and its goodness-of-fit is demonstrated. In addition to, comparisons to other models are implemented to show the flexibility of the presented model.
\end{abstract}

\section{Keywords}

Rayleigh Distribution, Truncated Weibull G-Family, Moments, Entropies, Quantile Function, Order Statistic, Maximum Likelihood Estimator

\section{Introduction}

Rayleigh distribution is used as a life time model and has applications in several fields such as survival analysis, reliability theory, and specially communication engineering. This distribution is a special case of two parameter Weibull distribution when the value of the shape parameter equals two. The Rayleigh distribution was originally derived by Rayleigh [2]. Johnson and balakrishnan [3] got continuous univariate distribution. Siddiqui [4] introduced the Rayleigh amplitude distribution the asymptotic distribution of a two-dimensional random walk. Howlader and Hossain [5] introduced bayes estimators for the scale parameter 
and the reliability function under the case of Type-II censored sampling data. Abd Elfattah et al. [6] studied maximum likelihood estimates efficiency of the parameter in three cases, called, Type-I, Type-II and progressive Type-II censored sampling schemes. Hendi et al. [7] introduced Bayes estimators of the scale parameter, reliability function and failure rate. Dey and Das [8] studied bayesian predictive intervals of the parameter of Rayleigh distribution. Dey [9] introduced bayes estimators for the parameter and reliability function of the Rayleigh distribution with different loss function. Soliman et al. [10] introduced type II half logistic family of distributions under application. Ferreira et al. [11] introduced concepts for the censored Rayleigh model by considering new loss function, called the Al-Bayyati Loss $(\mathrm{ABL})$ and comparing it to other known outcome. Al Mayali [12] compared some estimators of Rayleigh distribution with simulation. Singh and Srivastava [13] developed Bayesian estimation of parameter of inverse maxwell distribution via size-biased sampling.

The probability density function (p.d.f.) and cumulative distribution function (c.d.f.) of the Rayleigh distribution are given, respectively, by

$$
G(x ; \sigma)=1-\mathrm{e}^{\frac{x^{x^{2}}}{2 \sigma^{2}}}, x \geq 0, \sigma>0
$$

and

$$
g(x ; \sigma)=\frac{x}{\sigma^{2}} \mathrm{e}^{\frac{-x^{2}}{2 \sigma^{2}}}, x \geq 0, \sigma>0
$$

where $\sigma$ is an inverse scale parameter. From Equation (2), we obtain immediately the expected value (the mean) and the variance of a Rayleigh distribution of $X$ to be

$$
E(X)=\sigma \frac{\sqrt{\Pi}}{2}
$$

and

$$
\operatorname{Var}(X)=\sigma^{2}\left(\frac{4-\Pi}{2}\right)
$$

Weibull distribution plays an effective role in Reliability Theory. In fact, Weibull distribution is the first candidate for modeling cases with monotone hazard rates but it is not a good candidate for modeling cases without monotone hazard rates. Thus, we can see many researchers attempting to modify the Weibull distribution for applying under nonmonotone hazard rates. The truncated Weibull distribution is one of such distributions with bathtub shaped hazard rate function. It has been applied in several engineering areas, partially since it has more flexible hazard rate than Weibull distribution.

In the last few years, many researchers are interested to expand generating family in order to get better fit for data analyzing. Eugene and Famoye [14] introduced Beta-normal distribution. Cordeiro and de Castro [15] introduced new family generalized distribution. Ardianti [16] studied estimating parameter of 
Rayleigh distribution by using maximum likelihood method and bayes method. Cordeiro et al. [17] studied the exponentiated generalized class of distributions. Alzaatreh and Famoye [18] introduced a new method for generating families of continuous distributions. Bourguignon et al. [19] studied the Weibull-g family of probability distributions. Cordeiro et al. [20] studied Lomax-G. Cordeiro et al. [21] introduced the beta odd log-logistic generalized-G. Cordeiro et al. [22] introduced the exponentiated half-logistic family of distributions: Properties and applications. Hassan and Elgarhy [23] studied Kumaraswamy Weibull-G. Hassan and Hemeda [24] studied additive Weibull-G family of probability distribution. Elgarhy et al. [25] introduced exponentiated extended-G. Hassan et al. [26] studied the generalized additive weibull-g family of distributions. Hassan et al. [27] studied [0,1] truncated Fréchet-G. Abid and Abdulrazak [28] introduced truncated fréchet-g generator of distributions. Mansoor et al. [29] introduced a new familly of distributions to analyze lifetime data. Haq and Elgarhy [30] introduced the odd Fréchet-G family of probability distributions. Hassan and Nassr [31] studied Power lindley-g family of distributions.

Over the last two decades, several extensions of the well-known lifetime distributions have been developed for modeling many types of practical data sets. This development is followed by many approaches for generating new families of (probability) distributions which increase chances of modeling data of various random nature. Among those families, we can mention: Zografos and Balakrishnan [32] introduced the gamma-G (type 1). Ristic and Balakrishnan [33] studied the gamma-G (type 2). Saboor et al. [34] studied beta weighted modified Weibull distribution using the beta generator. Alizadeh et al. [35] presented the generalized transmuted family of distributions. Alizadeh et al. [36] show the odd-Burr generalized family of distributions. Jamal et al. [37] studied the odd Burr-III family of distributions. Bakouch et al. [38] introduced the extended odd family of probability distributions. In practical life problems, truncation arises in many fields, such as industry, biology, hydrology, reliability theory and medicine. An example of truncation is the progression of a disease which is not an increasing function, but will stabilize after time point. This point is called the truncation for the support of the variable of the interest which may be time, length, height etc. Therefore, many researchers are attracted to analyze such truncated data using truncated versions of the standard statistical distributions. For instance, the 1 truncated Weibull distribution has been applied to analyze the tree diameter and height distributions in forestry, fire size and high-cycle fatigue strength prediction (See Zhang and Xie [39]). Zaninetti and Ferraro [40] introduced the truncated Pareto distribution is compared to the Pareto distribution using astrophysics data and they concluded, generally, that the truncated Pareto distribution performs better than the Pareto. Recently, Burroughs and Tebbens [41] showed the suitability of truncated power law distributions for data sets of earthquake magnitudes and forest fire areas. Additional applications of the former distributions in hydrology and atmospheric science are given by Aban et al. [42]. 
In view of the work of [43], the advantages of the TW-G family are the following ones: 1) the corresponding functions have tractable expressions, including the quantile function; 2) there is only one additional parameter to the baseline distribution; 3 ) several members of this family enjoy interesting properties, including various shapes for the corresponding functions; 4) for some data sets, the TIW-G models can offer better alternatives to other well-established models, possibly defined with more parameters. In [43], these points are illustrated with the special member defined with the Rayleigh distribution as baseline. However, with a closer look on the shapes analysis, as well as platykurtic and high heavy tail properties for the pdf. With the idea in mind that the moderate lack of flexibility of the TW-G family can be improved, we explore the solution provided by Truncated Weibull Rayleigh (TW-R) distribution. Thus, it is obtained from TW-G families.

Therefore, we have a truncated general-G class of distributions and the only sub-model we aware of is the truncated Weibull -G family proposed by Najarzadegan et al. [1]. As a powerful alternative to beta-G family of distributions. We cannot investigate more analytic properties and therefore we aim to study extensively the truncated Weibull Rayleigh (TW-R) distribution to fit practical data from different domains and this is investigated in the application section, several distributions have been presented on an extension of known distributions. So, many methods for generating new distributions from classic ones were evolved. The TW-G family has the following cdf and pdf:

$$
F_{\mathrm{TW}-\mathrm{G}}(x ; \alpha, \beta)=A\left(1-\mathrm{e}^{-\alpha(G(x))^{\beta}}\right), x \in R, \alpha, \beta>0
$$

and

$$
f_{\text {TW-G }}(x ; \alpha, \beta)=A \alpha \beta g(x) G(x)^{\beta-1} \mathrm{e}^{-\alpha(G(x))^{\beta}}, x \in R, \alpha, \beta>0,
$$

where $A=\left(1-\mathrm{e}^{-\alpha}\right)^{-1}, \alpha$ is the scale parameter, $\beta$ is the shape parameter and $G$ is cdf of any baseline distribution.

The purpose of this paper is to show a new three parameter life time model relied on the TW-G family. The TW-R model gives more flexible model. We hope that the new model will interesting wide enforcements in several areas. This paper is presented as follows: In Section 2, the Truncated Weibull-Rayleigh is presented. Section 3 provides several mathematical properties of the TW-R distribution. The maximum likelihood method is carrieded out to get the estimators of the parameters in Section 4. Application to a real data explaining the execution of the new model is given in Section 5. Finally, show in Section 6.

A random variable is said to have the TW-R distribution by vector parameters $\Psi(\alpha, \beta, \sigma)$. If it has the following cdf and pdf:

$$
F_{\text {TW-R }}(x ; \Psi)=A\left[1-\exp \left(-\alpha\left(1-\mathrm{e}^{-\frac{x^{2}}{2 \sigma^{2}}}\right)^{\beta}\right)\right]
$$


and

$$
f_{\mathrm{TW}-\mathrm{R}}(x ; \Psi)=A \alpha \beta \sigma^{-2} x \mathrm{e}^{-\frac{x^{2}}{2 \sigma^{2}}}\left(1-\mathrm{e}^{-\frac{x^{2}}{2 \sigma^{2}}}\right)^{\beta-1} \exp \left(-\alpha\left(1-\mathrm{e}^{-\frac{x^{2}}{2 \sigma^{2}}}\right)^{\beta}\right),
$$

where $\alpha, \beta$ and $\sigma$ are shape and inverse scale parameter, respectively (Figure 1 and Figure 2).

From Equations (7) and (8), we obtain immediately the reliability function, the hazard function, reversed hazard rate function, cumulative hazard rate function and the Quantile function of truncated weibull rayleigh distribution.

The reliability function represents the probability of survival, giving by (Figure 3).

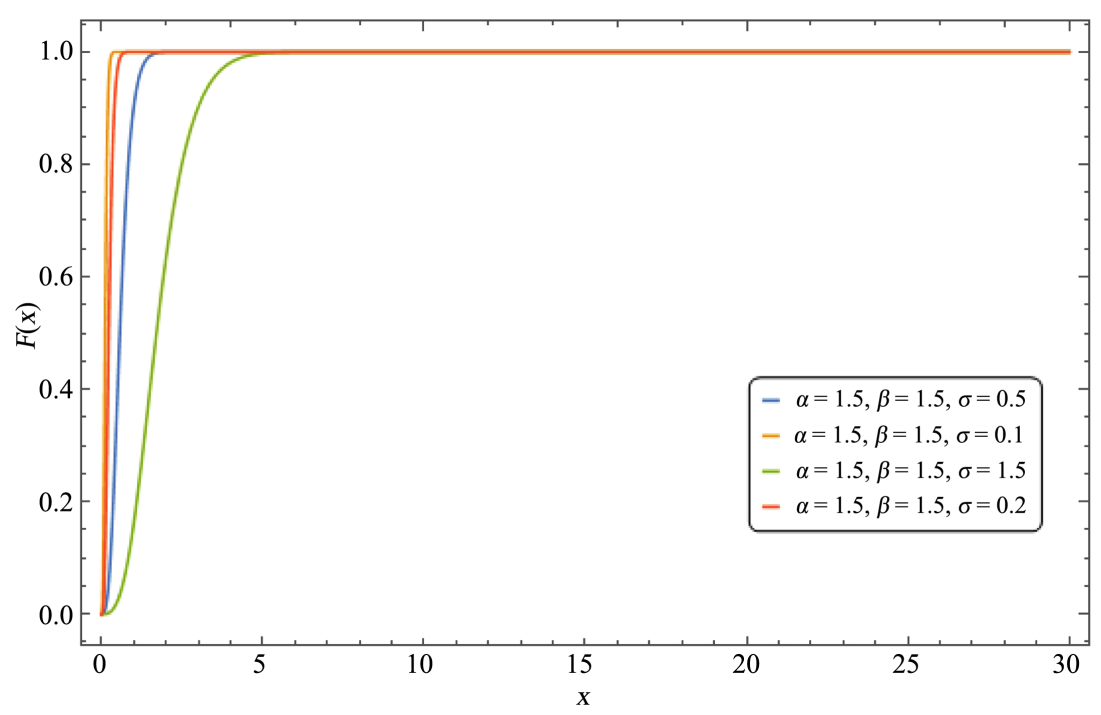

Figure 1. Plot of cdf of TW-R distribution.

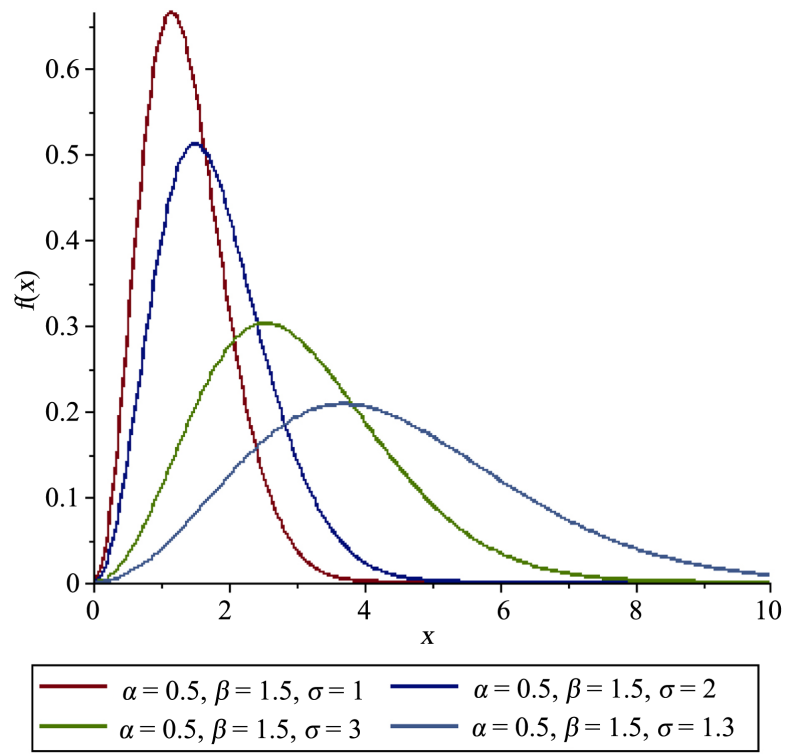

Figure 2. Plot of pdf of TW-R distribution. 


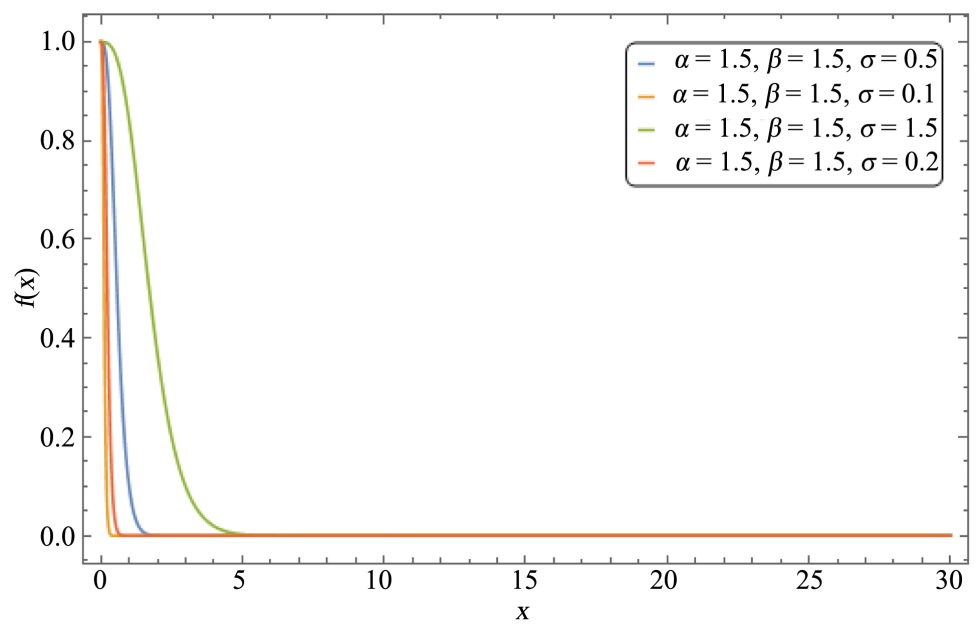

Figure 3. Plot of the reliability function of TW-R distribution.

$$
\bar{F}_{\mathrm{TW}-\mathrm{R}}(x ; \Psi)=1-F_{\mathrm{TW}-\mathrm{R}}(x ; \Psi)=1-A\left[1-\exp \left(-\alpha\left(1-\mathrm{e}^{-\frac{x^{2}}{2 \sigma^{2}}}\right)\right)^{\beta}\right] .
$$

The hazard rate function $h(x)$, reversed hazard rate function $h(x)$ and cumulative hazard rate function $H(x)$ are an important quantity characterizing life phenomena (Figure 4).

$$
\begin{aligned}
h_{\mathrm{TW}-\mathrm{R}}(x ; \Psi) & =\frac{f_{\mathrm{TW}-\mathrm{R}}(x ; \Psi)}{F_{\mathrm{TW}-\mathrm{R}}(x ; \Psi)} \\
= & \frac{A \alpha \beta \sigma^{-2} x \mathrm{e}^{-\left(\frac{x^{2}}{2 \sigma^{2}}\right)}\left(1-\mathrm{e}^{-\frac{x^{2}}{2 \sigma^{2}}}\right)^{\beta-1} \exp \left(1-\alpha \mathrm{e}^{\left.-\frac{x^{2}}{2 \sigma^{2}}\right)^{\beta}}\right.}{1-A\left[1-\exp \left(-\alpha\left(1-\mathrm{e}^{-\frac{x^{2}}{2 \sigma^{2}}}\right)^{\beta}\right)\right]} .
\end{aligned}
$$

Reversed hazard rate function is giving by

$$
\begin{aligned}
r_{\mathrm{TW}-\mathrm{R}}(x ; \Psi) & =\frac{f_{\mathrm{TW}-\mathrm{R}}(x ; \Psi)}{\bar{F}_{\mathrm{TW}-\mathrm{R}}(x ; \Psi)} \\
= & \frac{A \alpha \beta \sigma^{-2} x \mathrm{e}^{-\frac{x^{2}}{2 \sigma^{2}}}\left(1-\mathrm{e}^{-\frac{x^{2}}{2 \sigma^{2}}}\right)^{\beta-1} \exp \left(1-\alpha \mathrm{e}^{\left.-\frac{x^{2}}{2 \sigma^{2}}\right)^{\beta}}\right.}{1-A\left[1-\exp \left(-\alpha\left(1-\mathrm{e}^{-\frac{x^{2}}{2 \sigma^{2}}}\right)^{\beta}\right)\right]} .
\end{aligned}
$$

Cumulative hazard rate function of truncated weibull rayleigh distribution is giving by

$$
H_{\mathrm{TW}-\mathrm{R}}(x ; \Psi)=\frac{\mathrm{d}}{\mathrm{d} x}\left[h_{\mathrm{TW}-\mathrm{R}}(x ; \Psi)\right]=\ln \left[1-A\left[1-\exp \left(-\alpha\left(1-\mathrm{e}^{-\frac{x^{2}}{2 \sigma^{2}}}\right)^{\beta}\right)\right]\right] .
$$




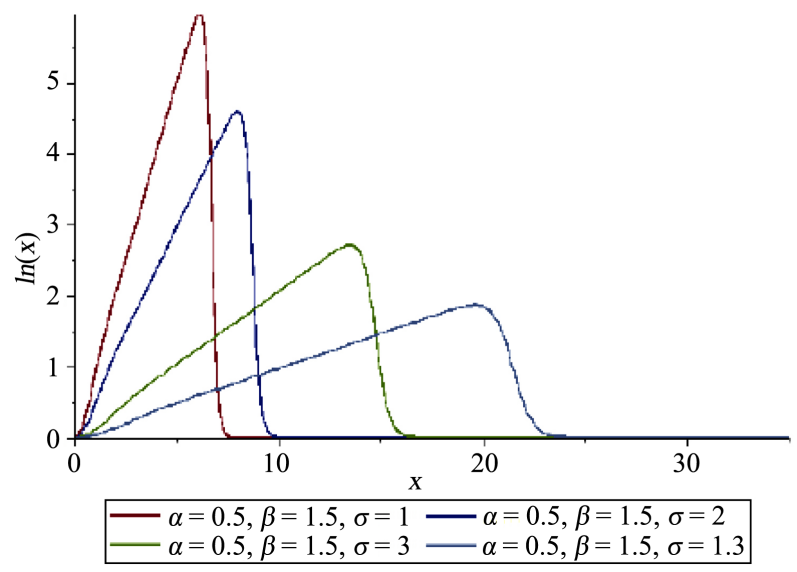

Figure 4. lot of the hazard function of TW-R distribution.

Additionally, the Quantile function of the TW-R can be produced by reversing cdf in Equation (7) as follows

$$
F_{\mathrm{TW}-\mathrm{R}}(x ; \Psi)=U,
$$

thus, we get

$$
x=\sqrt{-2 \sigma^{2} \ln \left[1-\left(\frac{-1}{\alpha} \ln \left(1-\frac{U}{A}\right)\right)^{\frac{1}{\beta}}\right]} .
$$

If $U$ a uniform variate in the unit interval $(0,1)$, then the random variable $X=Q(u)$ from follows Equation (7).

\section{Ordinary and Incomplete Moments}

Now, we obtain the $r^{\text {th }}$ moment about zero of $X$, say $E\left(X^{r}\right)$. From pdf in Equation (6), is giving

$$
E\left(X^{r}\right)=\Gamma\left(\frac{r}{2}+1\right) \sum_{i=0}^{\infty} \sum_{j=0}^{\infty} \frac{(-1)^{i+j}}{i !}\left(\begin{array}{c}
\beta(i+1)-1 \\
j
\end{array}\right) \frac{2^{r-1} \sigma^{2 r} \alpha^{i+1} \beta A}{(j+1)^{r+1}} .
$$

Proof

$$
E\left(X^{r}\right)=\int_{0}^{\infty} x^{r} f_{\mathrm{TW}-\mathrm{R}}(x ; \Psi) \mathrm{d} x
$$

then,

$$
\begin{aligned}
E\left(X^{r}\right) & =\int_{0}^{\infty} x^{r} A \alpha \beta \sigma^{-2} x \mathrm{e}^{-\left(\frac{x^{2}}{2 \sigma^{2}}\right)}\left(1-\mathrm{e}^{-\left(\frac{x^{2}}{2 \sigma^{2}}\right)}\right)^{\beta-1} \exp \left(-\alpha\left(1-\mathrm{e}^{-\frac{x^{2}}{2 \sigma^{2}}}\right)^{\beta}\right) \mathrm{d} x \\
& =\frac{A \alpha \beta}{\sigma^{2}} \int_{0}^{\infty} x^{r+1} \mathrm{e}^{-\left(\frac{x^{2}}{2 \sigma^{2}}\right)}\left(1-\mathrm{e}^{-\left(\frac{x^{2}}{2 \sigma^{2}}\right)}\right)^{\beta-1} \exp \left(-\alpha\left(1-\mathrm{e}^{-\frac{x^{2}}{2 \sigma^{2}}}\right)^{\beta}\right) \mathrm{d} x .
\end{aligned}
$$

By using exponential expansion, where exponential expansion is giving by equation 


$$
\mathrm{e}^{x}=\sum_{n=0}^{\infty} \frac{x^{n}}{n !}
$$

thus,

$$
\exp \left(-\alpha\left(1-\mathrm{e}^{-\frac{x^{2}}{2 \sigma^{2}}}\right)^{\beta}\right)=\sum_{n=0}^{\infty} \frac{(-1)^{i} \alpha^{i}\left(1-\mathrm{e}^{-\frac{x^{2}}{2 \sigma^{2}}}\right)^{i \beta}}{i !},
$$

then

$$
E\left(x^{r}\right)=\sum_{i=0}^{\infty} \frac{(-1)^{i}}{i !} \frac{A \alpha^{i+1} \beta}{\sigma^{2}} \int_{0}^{\infty} x^{r+1} \mathrm{e}^{-\left(\frac{x^{2}}{2 \sigma^{2}}\right)}\left(1-\mathrm{e}^{-\frac{x^{2}}{2 \sigma^{2}}}\right)^{\beta(i+1)-1} \mathrm{~d} x .
$$

By using binomial expansion, where binomial expansion is giving by equation

$$
(a+b)^{n}=\sum_{k=0}^{n}\left(\begin{array}{l}
n \\
k
\end{array}\right) a^{k} b^{n-k}
$$

thus,

$$
\begin{gathered}
\left(1-\mathrm{e}^{-\frac{x^{2}}{2 \sigma^{2}}}\right)^{\beta(i+1)-1}=\sum_{j=0}^{\beta(i+1)-1}(-1)^{j}\left(\begin{array}{c}
\beta(i+1)-1 \\
j
\end{array}\right) \mathrm{e}^{-j\left(\frac{x^{2}}{2 \sigma^{2}}\right)}, \\
E\left(x^{r}\right)=\sum_{i=0}^{\infty} \sum_{j=0}^{\infty} \frac{(-1)^{i+j}}{i !}\left(\begin{array}{c}
\beta(i+1)-1 \\
j
\end{array}\right) \frac{A \alpha^{i+1} \beta}{\sigma^{2}} \int_{0}^{\infty} x^{r+1} \mathrm{e}^{-(j+1)\left(\frac{x^{2}}{2 \sigma^{2}}\right)} \mathrm{d} x,
\end{gathered}
$$

Thus, the computation of this integration

$$
\int_{0}^{\infty} x^{r+1} e^{-(j+1)\left(\frac{x^{2}}{2 \sigma^{2}}\right)} \mathrm{d} x=\frac{1}{2}\left(\frac{2 \sigma^{2}}{j+1}\right)^{r+1} \Gamma\left(\frac{r}{2}+1\right) .
$$

This completes the proof.

\section{The Probability Weighted Moments (PWMs)}

The class of probability weighted moments (PWMs) is primarily used in estimating the parameters of a distribution whose inverse cannot be expressed explicitly. For a random variable $X$, its PWM giving by $\Upsilon_{r, s}$ is defined as

$$
\Upsilon_{r, s}=E\left[x^{r}(F(x))^{s}\right]=\int_{-\infty}^{\infty} x^{r} f(x)(F(x))^{s} .
$$

The probability weighted moments of the Truncated Weibull of rayleigh distribution is giving by:

$$
\begin{aligned}
\Upsilon_{r, s}= & \sum_{j=0}^{s} \sum_{k=0}^{\infty} \sum_{i=0}^{(k+1) \beta-1} \frac{(-1)^{i+j+k} \beta \alpha^{k+1} \sigma^{-2} A^{s+1}(j+1)^{k}}{k !}\left(\begin{array}{c}
(k+1) \beta-1 \\
i
\end{array}\right)\left(\begin{array}{l}
s \\
j
\end{array}\right) \\
& \times \frac{1}{2}\left(\frac{2 \sigma^{2}}{i+1}\right)^{\frac{r}{2}+1} \Gamma\left(\frac{r}{2}+1\right) .
\end{aligned}
$$

Proof 


$$
\Upsilon_{r, s}=\int_{-\infty}^{\infty} x^{r} f(x)(F(x))^{s},
$$

From Equations (7) and (8), we get

$$
\begin{aligned}
\Upsilon_{r, s}= & \int_{-\infty}^{\infty} x^{r+1} A \alpha \beta \sigma^{-2} \mathrm{e}^{-\left(\frac{x^{2}}{2 \sigma^{2}}\right)}\left(1-\mathrm{e}^{-\left(\frac{x^{2}}{2 \sigma^{2}}\right)}\right)^{\beta-1} \exp \left[-\alpha\left(1-\mathrm{e}^{-\left(\frac{x^{2}}{2 \sigma^{2}}\right)}\right)^{\beta}\right] \\
& \times\left[A\left[1-\exp \left[-\alpha\left(1-\mathrm{e}^{-\left(\frac{x^{2}}{2 \sigma^{2}}\right)}\right)\right]\right)^{\beta}\right] \mathrm{d} x .
\end{aligned}
$$

By using binomial expansion.

Thus,

$$
\left[1-\exp \left(-\alpha\left(1-\mathrm{e}^{-\left(\frac{x^{2}}{2 \sigma^{2}}\right)}\right)^{\beta}\right)\right]^{s}=\sum_{j=0}^{s}(-1)^{j}\left(\begin{array}{l}
s \\
j
\end{array}\right) \exp \left[-j \alpha\left(1-\mathrm{e}^{-\frac{x^{2}}{2 \sigma^{2}}}\right)^{\beta}\right],
$$

By substitution in Equation (8). We get,

$$
\begin{aligned}
\Upsilon_{r, s}= & \sum_{j=0}^{s}(-1)^{j}\left(\begin{array}{c}
s \\
j
\end{array}\right) \int_{0}^{\infty} x^{r+1} A^{s+1} \alpha \beta \sigma^{-2} \mathrm{e}^{-\left(\frac{x^{2}}{2 \sigma^{2}}\right)}\left(1-\mathrm{e}^{-\left(\frac{x^{2}}{2 \sigma^{2}}\right)}\right)^{\beta-1} \\
& \times \exp \left[-\alpha(j+1)\left(1-\mathrm{e}^{-\frac{x^{2}}{2 \sigma^{2}}}\right)^{\beta}\right] \mathrm{d} x .
\end{aligned}
$$

By using exponential expansion

$$
\begin{gathered}
\exp \left[-\alpha(j+1)\left(1-\mathrm{e}^{-\frac{x^{2}}{2 \sigma^{2}}}\right)^{\beta}\right]=\sum_{k=0}^{\infty} \frac{(-1)^{k} \alpha^{k}(j+1)^{k}\left(1-\mathrm{e}^{-\frac{x^{2}}{2 \sigma^{2}}}\right)^{k \beta}}{k !}, \\
\Upsilon_{r, s}=\sum_{j=0}^{s} \sum_{k=0}^{\infty} \frac{(-1)^{j+k} \beta \alpha^{k+1} \sigma^{-2} A^{s+1}(j+1)^{k}}{k !}\left(\begin{array}{l}
s \\
j
\end{array}\right) \int_{0}^{\infty} x^{r+1} \mathrm{e}^{-\left(\frac{x^{2}}{2 \sigma^{2}}\right)}\left(1-\mathrm{e}^{-\frac{x^{2}}{2 \sigma^{2}}}\right)^{(k+1) \beta-1} \mathrm{~d} x .
\end{gathered}
$$

By using binomial expansion

$$
\left(1-\mathrm{e}^{-\frac{x^{2}}{2 \sigma^{2}}}\right)^{(k+1) \beta-1}=\sum_{i=0}^{(k+1) \beta-1}(-1)^{i}\left(\begin{array}{c}
(k+1) \beta-1 \\
i
\end{array}\right) \mathrm{e}^{-i\left(\frac{x^{2}}{2 \sigma^{2}}\right)},
$$

The computation of the following integration,

$$
\int_{0}^{\infty} x^{r+1} e^{-(i+1)\left(\frac{x^{2}}{2 \sigma^{2}}\right)} \mathrm{d} x=\frac{1}{2}\left(\frac{2 \sigma^{2}}{i+1}\right)^{\frac{r}{2}+1} \Gamma\left(\frac{r}{2}+1\right) .
$$

thus 


$$
\begin{aligned}
\Upsilon_{r, s}= & \sum_{i=0}^{(k+1) \beta-1} \sum_{j=0}^{s} \sum_{k=0}^{\infty} \frac{(-1)^{i+j+k} \beta \alpha^{k+1} \sigma^{-2} A^{s+1}(j+1)^{k}}{k !}\left(\begin{array}{c}
(k+1) \beta-1 \\
i
\end{array}\right)\left(\begin{array}{l}
s \\
j
\end{array}\right) \\
& \times \frac{1}{2}\left(\frac{2 \sigma^{2}}{i+1}\right)^{\frac{r}{2}+1} \Gamma\left(\frac{r}{2}+1\right) .
\end{aligned}
$$

This completes the proof.

\section{Rényi and q Entropies}

An entropy is a measure of variation or uncertainty of a random variable $X$. The Rényi entropy is defined by

$$
I_{R}(x)=\frac{1}{1-R} \log \int_{0}^{\infty} f(x)^{R} \mathrm{~d} x, R>0 \text { and } R \neq 0 .
$$

Now, we will get Rényi entropy of Truncated Weibull Rayleigh distribution (TW-R) is giving by the following theorem

$$
I_{\mathrm{TW}-\mathrm{R}}(x, \Psi)=\frac{1}{2(1-R)} \log \sum_{i=0}^{\infty} \sum_{j=0}^{(R+i) \beta-R} \varepsilon_{i}\left(\frac{2 \sigma^{2}}{R+j}\right)^{\frac{1}{2}(R+1)} \Gamma\left(\frac{1}{2}(R-1)\right) .
$$

Proof:

$$
I_{R}(x)=\frac{1}{1-R} \log \int_{0}^{\infty} f(x)^{R} \mathrm{~d} x .
$$

From Equation (8). We get,

$$
\begin{aligned}
I_{\mathrm{TW}-\mathrm{R}}(x, \Psi)= & \frac{1}{1-R} \log \int_{0}^{\infty} A^{R} \alpha^{R} \beta^{R} \sigma^{-2 R} x^{R} \mathrm{e}^{-\frac{R x^{2}}{2 \sigma^{2}}}\left(1-\mathrm{e}^{-\frac{x^{2}}{2 \sigma^{2}}}\right)^{R \beta-R} \\
& \times \exp \left(-\alpha R\left(1-\mathrm{e}^{-\frac{x^{2}}{2 \sigma^{2}}}\right)^{\beta}\right) \mathrm{d} x .
\end{aligned}
$$

By using exponential expansion,

$$
\exp \left(-\alpha R\left(1-\mathrm{e}^{-\frac{x^{2}}{2 \sigma^{2}}}\right)^{\beta}\right)=\sum_{i=0}^{\infty} \frac{(-1)^{i}(\alpha)^{i}(R)^{i}\left(1-\mathrm{e}^{-\frac{x^{2}}{2 \sigma^{2}}}\right)^{i \beta}}{i !},
$$

thus,

$$
\begin{aligned}
& I_{\mathrm{TW}-\mathrm{R}}(x, \Psi) \\
& =\frac{1}{1-R} \log \left[\sum_{i=0}^{\infty} \frac{(-1)^{i}(\alpha)^{i}(R)^{i}}{i !}\left(\frac{A \alpha \beta}{\sigma^{2}}\right)^{R} \int_{0}^{\infty} x^{R} \mathrm{e}^{-R\left(\frac{x^{2}}{2 \sigma^{2}}\right)}\left(1-\mathrm{e}^{-\frac{x^{2}}{2 \sigma^{2}}}\right)^{(R+i) \beta-R} \mathrm{~d} x\right],
\end{aligned}
$$

By using binomial expansion

$$
\left(1-\mathrm{e}^{-\frac{x^{2}}{2 \sigma^{2}}}\right)^{(R+i) \beta-R}=\sum_{j=0}^{(R+i) \beta-R}(-1)^{j}\left(\begin{array}{c}
(R+i) \beta-R \\
j
\end{array}\right) \mathrm{e}^{-j \frac{x^{2}}{2 \sigma^{2}}},
$$


thus

$$
\begin{gathered}
I_{\mathrm{TW}-\mathrm{R}}(x, \Psi)=\frac{1}{1-R} \log \left[\sum_{i=0}^{\infty} \sum_{j=0}^{(R+i) \beta-R} \frac{(-1)^{i+j}(\alpha)^{i}(R)^{i}}{i !}\left(\frac{A \alpha \beta}{\sigma^{2}}\right)^{R}\left(\begin{array}{c}
(R+i) \beta-R \\
j
\end{array}\right)\right. \\
\left.\quad \times \int_{0}^{\infty} x^{R} \mathrm{e}^{-(R+j)\left(\frac{x^{2}}{2 \sigma^{2}}\right)} \mathrm{d} x\right] . \\
\text { Let } \varepsilon_{i}=\frac{(-1)^{i+j}(\alpha)^{i}(R)^{i}}{i !}\left(\frac{A \alpha \beta}{\sigma^{2}}\right)^{R}\left(\begin{array}{c}
(R+i) \beta-R \\
j
\end{array}\right), \\
I_{\mathrm{TW}-\mathrm{R}}(x, \Psi)=\frac{1}{1-R} \log \left[\sum_{i=0}^{\infty} \sum_{j=0}^{(R+i) \beta-R} \varepsilon_{i} \int_{0}^{\infty} x^{R} \mathrm{e}^{-(R+j)\left(\frac{x^{2}}{2 \sigma^{2}}\right)} \mathrm{d} x\right] .
\end{gathered}
$$

The computation of the following integration,

$$
\int_{0}^{\infty} x^{R} \mathrm{e}^{-(R+j)\left(\frac{x^{2}}{2 \sigma^{2}}\right)} \mathrm{d} x=\frac{1}{2}\left(\frac{2 \sigma^{2}}{R+j}\right)^{\frac{1}{2}(R+1)} \Gamma\left(\frac{1}{2}(R-1)\right) .
$$

This completes the proof.

\section{Order Statistics}

Let $x_{1}, x_{2}, \cdots, x_{n}$ be independent and identically distributed random variables with continuous distribution function $F(x)$, is given by

$$
f_{X(r)}(x)=\frac{1}{B(r, n-r+1)} f(x)[F(x)]^{r-1}[1-F(x)]^{n-r} .
$$

By using binomial expansion,

$$
f_{X(r)}(x)=\frac{f(x)}{B(r, n-r+1)} \sum_{j=0}^{n-r}(-1)^{j}\left(\begin{array}{c}
n-r \\
j
\end{array}\right)[F(x)]^{j+r-1} .
$$

By substituting Equations (8) and (7) in Equation (20) and using binomial expansion then,

$$
\begin{aligned}
f_{X(r)}(x)= & \frac{1}{B(r, n-r+1)} \sum_{j=0}^{n-r}(-1)^{j}\left(\begin{array}{c}
n-r \\
j
\end{array}\right) A^{j+r-1}\left(1-\exp \left[-\alpha\left(1-\mathrm{e}^{-\frac{x^{2}}{2 \sigma^{2}}}\right)^{\beta}\right]\right)^{j+r-1} \\
& \times A \alpha \beta \sigma^{-2} x \mathrm{e}^{-\left(\frac{x^{2}}{2 \sigma^{2}}\right)}\left(1-\mathrm{e}^{-\frac{x^{2}}{2 \sigma^{2}}}\right)^{\beta-1} \exp \left[-\alpha\left(1-\mathrm{e}^{-\frac{x^{2}}{2 \sigma^{2}}}\right)^{\beta}\right]
\end{aligned}
$$

where

$$
\left(1-\exp \left[-\alpha\left(1-\mathrm{e}^{-\frac{x^{2}}{2 \sigma^{2}}}\right)^{\beta}\right]\right)^{j+r-1}=\sum_{k=0}^{j+r-1}(-1)^{k}\left(\begin{array}{c}
j+r-1 \\
k
\end{array}\right) \exp \left[-\alpha k\left(1-\mathrm{e}^{-\frac{x^{2}}{2 \sigma^{2}}}\right)^{\beta}\right],
$$

by using exponential expansion, 


$$
\exp \left[-\alpha(k+1)\left(1-\mathrm{e}^{-\frac{x^{2}}{2 \sigma^{2}}}\right)^{\beta}\right]=\sum_{i=0}^{\infty} \frac{(-1)^{i}(k+1)^{i} \alpha^{i}\left(1-\mathrm{e}^{-\frac{x^{2}}{2 \sigma^{2}}}\right)^{i \beta}}{i !},
$$

thus,

$$
\begin{aligned}
f_{X(r)}(x)= & \frac{1}{B(r, n-r+1)} \sum_{v=0}^{n-r} \sum_{k=0}^{j+r-1} \sum_{i=0}^{\infty} \frac{(-1)^{j+k}}{i !}\left(\begin{array}{c}
n-r \\
j
\end{array}\right)\left(\begin{array}{c}
j+r-1 \\
k
\end{array}\right) \\
& \times A^{j+r} \alpha^{i+1} \beta \sigma^{-2}(k+1)^{i} x \mathrm{e}^{-\left(\frac{x^{2}}{2 \sigma^{2}}\right)}\left(1-\mathrm{e}^{-\frac{x^{2}}{2 \sigma^{2}}}\right)^{(i+1) \beta-1} .
\end{aligned}
$$

\section{Maximum Likehood Estimation}

In this section, we show estimation of the parameters of TW-R model by the maximum likelihood method. Assume $x_{1}, x_{2}, x_{3}, \cdots, x_{n}$ be random variables from truncated weibull rayleigh distribution with the set of parameter $\Psi(\alpha, \beta, \sigma)$. The likelihood function based on the observed random sample of size $n$ from Equation (8) is given by

$$
\begin{aligned}
& L(x, \Psi)=\prod_{i=1}^{n}\left(1-\mathrm{e}^{-\alpha}\right)^{-1} \alpha \beta \sigma^{-2} x_{i} \mathrm{e}^{-\left(\frac{x_{i}^{2}}{2 \sigma^{2}}\right)}\left(1-\mathrm{e}^{\left.-\left(\frac{x_{i}^{2}}{2 \sigma^{2}}\right)\right)^{\beta-1}} \exp \left(-\alpha\left(1-\mathrm{e}^{\left.\left.-\left(\frac{x^{2}}{2 \sigma^{2}}\right)\right)^{\beta}\right)}\right.\right.\right. \\
& =\left(1-\mathrm{e}^{-\alpha}\right)^{-n} \alpha^{n} \beta^{n} \sigma^{-2 n} \prod_{i=1}^{n} x_{i} \mathrm{e}^{-\sum_{i=0}^{n} \frac{x_{i}^{2}}{2 \sigma^{2}}} \prod_{i=1}^{n}\left(1-\mathrm{e}^{\left.-\left(\frac{x_{i}^{2}}{2 \sigma^{2}}\right)\right)^{\beta-1}} \exp \left(-\alpha\left(1-\mathrm{e}^{-\frac{x_{i}^{2}}{2 \sigma^{2}}}\right)\right)^{\beta} .\right.
\end{aligned}
$$

By taking $\ln$ function on both sides,

$$
\begin{aligned}
\ell(x, \Psi)= & -n \ln \left[1-\mathrm{e}^{-\alpha}\right]+n \ln \alpha+n \ln \beta-2 n \ln \sigma+\sum_{i=0}^{n} \ln x_{i}-\frac{\sum_{i=0}^{n} x_{i}^{2}}{2 \sigma^{2}} \\
& +(\beta-1) \sum_{i=0}^{n} \ln \left(1-\mathrm{e}^{-\frac{x_{i}^{2}}{2 \sigma^{2}}}\right)-\alpha \sum_{i=0}^{n}\left(1-\mathrm{e}^{-\frac{x_{i}^{2}}{2 \sigma^{2}}}\right)^{\beta} .
\end{aligned}
$$

The first derivatives of $\ell(x, \Psi)$ with respect to the parameters $\alpha, \beta$ and $\sigma$ are

$$
\begin{gathered}
\frac{\partial \ell}{\partial \alpha}=\frac{-\alpha n \mathrm{e}^{-\alpha}}{1-\mathrm{e}^{-\alpha}}+\frac{n}{\alpha}-\sum_{i=0}^{n}\left(1-\mathrm{e}^{\left.-\left(\frac{x_{i}^{2}}{2 \sigma^{2}}\right)\right)^{\beta}},\right. \\
\frac{\partial \ell}{\partial \beta}=\frac{n}{\beta}+\sum_{i=0}^{n} \ln \left(1-\mathrm{e}^{-\frac{x_{i}^{2}}{2 \sigma^{2}}}\right)-\alpha \sum_{i=0}^{n}\left(1-\mathrm{e}^{-\frac{x_{i}^{2}}{2 \sigma^{2}}}\right)^{\beta} \ln \left(1-\mathrm{e}^{-\frac{x_{i}^{2}}{2 \sigma^{2}}}\right)
\end{gathered}
$$

and 


$$
\begin{aligned}
\frac{\partial \ell}{\partial \sigma}= & \frac{-2 n}{\sigma}+\frac{1}{\sigma^{3}} \sum_{i=0}^{n} x_{i}^{2}-\frac{\beta-1}{\sigma^{3}} \sum_{i=0}^{n} x_{i}^{2} \mathrm{e}^{-\frac{x_{i}^{2}}{2 \sigma^{2}}}\left[1-\mathrm{e}^{-\left(\frac{x_{i}^{2}}{2 \sigma^{2}}\right)}\right]^{-1} \\
& -\frac{\alpha \beta}{\sigma^{3}} \sum_{i=0}^{n} x_{i}^{2} \mathrm{e}^{-\frac{x_{i}^{2}}{2 \sigma^{2}}}\left[1-\mathrm{e}^{-\frac{x_{i}^{2}}{2 \sigma^{2}}}\right]^{\beta-1} .
\end{aligned}
$$

Therefore, solutions of these equations simultaneously yeild the MLE $\ell(x, \Psi)$ of parameters, unfortunately these equations cannot be solved analytically and numerical iterative methods can be employed to solve them. For interval estimation and hypothesis testings under the parameters, we require the information matrix $I(\Theta)=\left[-\frac{\partial^{2} \ln (\Theta)}{\partial \Theta^{2}}\right]$,

$$
I(\Theta)=\frac{\partial^{2} \ln (\Theta)}{\partial \Theta^{2}}=\left[\begin{array}{lll}
I_{11} & I_{12} & I_{13} \\
I_{21} & I_{22} & I_{23} \\
I_{31} & I_{32} & I_{33}
\end{array}\right],
$$

whose elements are

$$
\begin{aligned}
& I_{11}=\frac{\partial^{2} \ell}{\partial \alpha^{2}}=\frac{-n \mathrm{e}^{-\alpha}}{1-\mathrm{e}^{-\alpha}}+\frac{n \alpha \mathrm{e}^{-\alpha}}{\left(1-\mathrm{e}^{-\alpha}\right)^{2}}+\frac{-n}{\alpha^{2}}-n \ln \left[1-\mathrm{e}^{-\alpha}\right]=0, \\
& I_{22}=\frac{\partial^{2} \ell}{\partial \beta^{2}}=\frac{-n}{\beta^{2}}-\alpha \sum_{i=0}^{n}\left(1-\mathrm{e}^{-\left(\frac{x_{i}^{2}}{2 \sigma^{2}}\right)}\right)^{\beta} \ln \left(1-\mathrm{e}^{-\left(\frac{x_{i}^{2}}{2 \sigma^{2}}\right)}\right)=0 \text {, } \\
& I_{33}=\frac{\partial^{2} \ell}{\partial \sigma^{2}} \\
& =\frac{2 n}{\sigma^{2}}+\frac{3}{\sigma^{3}} \sum_{i=0}^{n} x_{i}^{2}-(\beta-1) \sum_{i=0}^{n}\left[x_{i}^{2}\left(1-\mathrm{e}^{-\frac{x_{i}^{2}}{2 \sigma^{2}}}\right)-\left(3-2 x_{i}^{2}\right) \sigma^{-4} \mathrm{e}^{-\frac{x_{i}^{2}}{2 \sigma^{2}}}\right]\left(1-\mathrm{e}^{-\frac{x_{i}^{2}}{2 \sigma^{2}}}\right)^{-2} \\
& -\alpha \beta \sum_{i=0}^{n} x_{i}^{2}\left[-3 \sigma^{-4}-\sigma^{2} x_{i}^{2}+(\beta-1) \sigma^{-1} x_{i}^{2}\left(1-\mathrm{e}^{-\frac{x_{i}^{2}}{2 \sigma^{2}}}\right)^{-1}\right] \mathrm{e}^{-\frac{x_{i}^{2}}{2 \sigma^{2}}}\left(1-\mathrm{e}^{-\frac{x_{i}^{2}}{2 \sigma^{2}}}\right)^{\beta-1} \\
& =0 \text {, } \\
& \begin{array}{l}
I_{12}=I_{21}=\frac{\partial^{2} \ell}{\partial \alpha \partial \beta}=-\sum_{i=0}^{n}\left(1-\mathrm{e}^{-\frac{x_{i}^{2}}{2 \sigma^{2}}}\right)^{\beta} \ln \left(1-\mathrm{e}^{-\frac{x_{i}^{2}}{2 \sigma^{2}}}\right)=0, \\
I_{13}=I_{31}=\frac{\partial^{2} \ell}{\partial \alpha \partial \sigma}=-\frac{\beta}{\sigma^{3}} \sum_{i=0}^{n} x_{i}^{2} \mathrm{e}^{-\frac{x_{i}^{2}}{2 \sigma^{2}}}\left[1-\mathrm{e}^{-\frac{x_{i}^{2}}{2 \sigma^{2}}}\right]^{\beta-1}=0
\end{array}
\end{aligned}
$$

and

$$
\begin{aligned}
I_{23} & =I_{32}=\frac{\partial^{2} \ell}{\partial \beta \partial \sigma} \\
& =\frac{2 n}{\beta^{3}}-\frac{\alpha \beta}{\sigma} \sum_{i=0}^{n} x_{i}^{2}\left(1-\mathrm{e}^{-\frac{x_{i}^{2}}{2 \sigma^{2}}}\right)^{\beta-1} \ln \left(1-\mathrm{e}^{-\frac{x_{i}^{2}}{2 \sigma^{2}}}\right)-\sum_{i=0}^{n} \sigma^{-1} x_{i}^{2}\left(1-\mathrm{e}^{-\frac{x_{i}^{2}}{2 \sigma^{2}}}\right)^{\beta-1}=0 .
\end{aligned}
$$




\section{Application}

In this section, we introduce an application to a real data set to evaluate the flexibility of the the truncated weibull Rayleigh (TW-R) model. We compare the fits of the TW-R distribution with the Truncated Weibull Fréchet (TWFr), type II Topp Leone inverse Rayleigh (TIITLIR) and inverse Rayleigh (IR) distributions. The data set is got from Mansoor et al. [29] expresss failure times of 63 airbcraft Windshield. The data are:

0.0461 .4362 .5920 .1401 .4922 .6000 .1501 .5802 .6700 .2482 .878

1.7192 .7171 .7942 .8190 .3131 .9152 .8200 .3890 .2801 .9200 .487

1.9632 .9500 .6221 .9783 .0030 .9002 .0533 .1020 .9522 .0654 .015

3.3040 .9962 .1173 .4831 .0032 .1373 .5001 .0102 .1413 .622

1.0852 .1633 .6651 .0922 .1833 .6951 .1522 .2401 .1832 .341

4.6281 .2442 .4354 .8061 .2492 .4644 .8811 .2622 .5435 .140 .

In Table 1, the analytical measures including, Akaike information criterion (AIC), the Bayesian information criterion (BIC), and Kolmogorov-Smirnov $(k-s)$ are introduced. Table 1 list the MLEs of the model parameters and their corresponding standard where as AIC, BIC and $k$-s.

The new generated Truncated Weibull Rayleigh distribution (TW-R) is compared with the Truncated Weibull Fréchet (TWFr), type II Topp Leone inverse Rayleigh (TIITLIR) and inverse Rayleigh (IR) distributions. For these data sets. The new distribution is compared with other distributions. By using some numerous fineness of fit measures such as the log likelihood function $(-2 \log \mathrm{L})$, Akaike Information Criterion (AIC), Bayesian Information Criterion (BIC), the Bayesian information criterion (BIC), and Kolmogorov-Smirnov $(k-s)$. The assessment parameters and fineness of fit measures of the pervious real life data sets. The fineness of fit measures such as $-2 \operatorname{LogL}$, AIC, BIC and for the $(k-s)$.

From the obtained results are presented in Table 1. As we can see from the results, the Truncated Weibull Rayleigh distribution is strong competitor to the compared models TWFr, TIITLIR and IR. Moreover, among all compared models, the TW-R distribution has the smallest values of the AIC, $-2 \operatorname{LogL}, \mathrm{BIC}$, and $k$-s. Thus, we can conclude that the TW-R distribution is the best fit among those models. Figure 5 display the plot of the fitted model for the data sets. This figure show the best fit of TW-R distribution.

Table 1. MLEs of the parameters, $-2 \log$, AIC, BIC and for the $(k-s)$ data set.

\begin{tabular}{ccccc}
\hline Distribution & $-2 \operatorname{LogL}$ & AIC & BIC & $k-s$ \\
\hline $\begin{array}{c}\text { TWR } \\
(\alpha, \beta, \sigma)\end{array}$ & 285.55 & 291.55 & 290.95 & 0.108456 \\
$\begin{array}{c}\text { TWFr } \\
(\alpha, \beta, \delta, \mu)\end{array}$ & 295.5 & 301.5 & 300.898 & 0.126968 \\
$\begin{array}{c}\text { TIITLIR } \\
(\alpha, \theta)\end{array}$ & 312.457 & 316.457 & 316.056 & 0.38516 \\
IR & & & & \\
$(\alpha)$ & 500.807 & 504.807 & 502.606 & 0.74246 \\
\hline
\end{tabular}




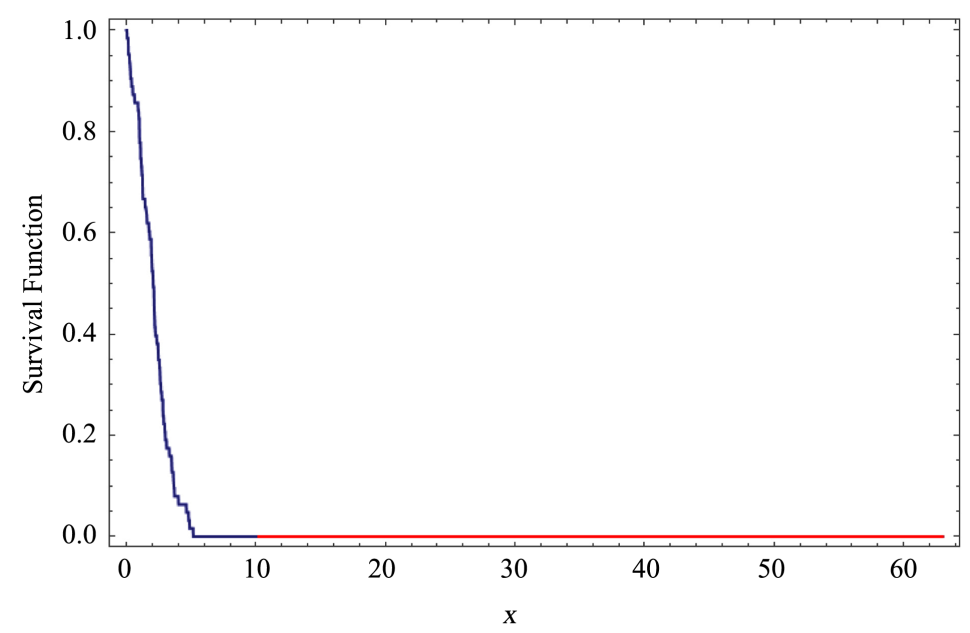

Figure 5. Plot of cdf of The estimated .tted models.

\section{Conclusion}

In statistical analysis many distributions are used to show sets of data. Recently, new distributions are derived to extend some of well-known families of distributions, such as the new distributions are more flexible than the others to model real data. The composing of some distributions with each other's in some way has been in the first of data modeling. In this paper, we introduced a new family of continuous distributions based on Truncated Weibull generating family. We introduce a new three parameters-model, called the truncated Weibull Rayleigh distribution. We derive expressions for the ordinary and incomplete moments, probability weighted moments. We discuss maximum likelihood estimation. Application shows that the Truncated Weibull Rayleigh distribution provides consistently better fit than other models.

\section{Conflicts of Interest}

The authors declare no conflicts of interest regarding the publication of this paper.

\section{References}

[1] Najarzadegan, H., Alamatsaz, M.H. and Hayati, S. (2017) Truncated Weibull-G More Flexible and More Reliable than Geta-G Distribution. International Journal of Statistics and Probability, 6, 1-17. https://doi.org/10.5539/ijsp.v6n5p1

[2] Rayleigh, L. (1880) XII. On the Resultant of a Large Number of Vibrations of the Same Pitch and of Arbitrary Phase. The London, Edinburgh, and Dublin Philosophical Magazine and Journal of Science, 10, 73-78. https://doi.org/10.1080/14786448008626893

[3] Johnson, N., Kotz, S. and Balakrishnan, N. (1994) Continuous Univariate Distributions. Wiley, Hoboken, 289, 607.

[4] Siddiqui, M.M. (1962) Some Problems Connected with Rayleigh Distributions. Journal of Research of the National Bureau of Standards, 66, 167-174. https://doi.org/10.6028/jres.066D.020 
[5] Howlader, H.A. and Hossain, A. (1995) On Bayesian Estimation and Prediction from Rayleigh Based on Type II Censored Data. Communications in Statistics-Theory and Methods, 24, 2251-2259. https://doi.org/10.1080/03610929508831614

[6] Abd Elfattah, A.M., Hassan, A.S. and Ziedan, D.M. (2006) Efficiency of Maximum Likelihood Estimators under Different Censored Sampling Schemes for Rayleigh Distribution. InterStat, Elctronic Journal, 1, 1-16.

[7] Hendi, M.L., Abu-Youssef, S.E. and Alraddadi, A.A. (2007) A Bayesian Analysis of Record Statistics from the Rayleigh Model. International Mathematical Forum, 2, 619-631. https://doi.org/10.12988/imf.2007.07057

[8] Dey, S. and Das, M.K. (2007) A Note on Prediction Interval for a Rayleigh Distribution: Bayesian Approach. American Journal of Mathematical and Management Sciences, 27, 43-48. https://doi.org/10.1080/01966324.2007.10737687

[9] Dey, S. (2009) Comparison of Bayes Estimators of the Parameter and Reliability Function for Rayleigh Distribution under Different Loss Functions. Malaysian Journal of Mathematical Sciences, 3, 247-264.

[10] Soliman, A.H., Elgarhy, M.A.E. and Shakil, M. (2017) Type II Half Logistic Family of Distributions with Applications. Pakistan Journal of Statistics and Operation Research, 13, 245-264. https://doi.org/10.18187/pjsor.v13i2.1560

[11] Ferreira, J.T., Bekker, A. and Arashi, M. (2016) Objective Bayesian Estimators for the Right Censored Rayleigh Distribution: Evaluating the Al-Bayyati Loss Function. REVSTAT-Statistical Journal, 14, 433-454. https://doi.org/10.4102/satnt.v33i1.1040

[12] Al Mayali, Y.M. (2013) A Comparison for Some of the Estimators of Rayleigh Distribution with Simulation. Journal of Kerbala University, 11, 104-112.

[13] Singh, K.L. and Srivastava, R.S. (2012) Bayesian Estimation of Parameter of Inverse Maxwell Distribution via Size-Biased Sampling. International Journal of Science and Research, 3, 2319-7064.

[14] Eugene, N., Lee, C. and Famoye, F. (2002) Beta-Normal Distribution and Its Applications. Communications in Statistics-Theory and Methods, 31, 497-512. https://doi.org/10.1081/STA-120003130

[15] Cordeiro, G.M. and de Castro, M. (2011) A New Family of Generalized Distributions. Journal of Statistical Computation and Simulation, 81, 883-898. https://doi.org/10.1080/00949650903530745

[16] Ardianti, F. (2018) Estimating Parameter of Rayleigh Distribution by Using Maximum Likelihood Method and Bayes Method. IOP Conference Series: Materials Science and Engineering, 300, Article ID: 012036. https://doi.org/10.1088/1757-899X/300/1/012036

[17] Cordeiro, G.M., Ortega, E.M. and da Cunha, D.C. (2013) The Exponentiated Generalized Class of Distributions. Journal of Data Science, 11, 1-27. https://doi.org/10.6339/JDS.201301_11(1).0001

[18] Alzaatreh, A., Lee, C. and Famoye, F. (2013) A New Method for Generating Families of Continuous Distributions. Metron, 71, 63-79. https://doi.org/10.1007/s40300-013-0007-y

[19] Bourguignon, M., Silva, R.B. and Cordeiro, G.M. (2014) The Weibull-G Family of Probability Distributions. Journal of Data Science, 12, 53-68.

https://doi.org/10.6339/JDS.201401_12(1).0004

[20] Cordeiro, G.M., Ortega, E.M., Popović, B.V. and Pescim, R.R. (2014) The Lomax Generator of Distributions: Properties, Minification Process and Regression Model. 
Applied Mathematics and Computation, 247, 465-486.

https://doi.org/10.1016/j.amc.2014.09.004

[21] Cordeiro, G.M., Alizadeh, M., Tahir, M.H., Mansoor, M., Bourguignon, M. and Hamedani, G.G. (2016) The Beta Odd Log-Logistic Generalized Family of Distributions. Hacettepe Journal of Mathematics and Statistics, 45, 1175-1202. https://doi.org/10.15672/HJMS.20157311545

[22] Cordeiro, G.M., Alizadeh, M. and Ortega, E.M. (2014) The Exponentiated Half-Logistic Family of Distributions: Properties and Applications. Journal of Probability and Statistics, 2014, Article ID: 864396. https://doi.org/10.1155/2014/864396

[23] Hassan, A.S. and Elgarhy, M. (2016) Kumaraswamy Weibull-Generated Family of Distributions with Applications. Advances and Application in Statistics, 48, 205-239. https://doi.org/10.17654/AS048030205

[24] Hassan, A.S. and Hemeda, S.E. (2016) The Additive Weibull-G Family of Probability Distributions. International Journal of Mathematics and Its Applications, 4, 151-164.

[25] Elgarhy, M., Haq, M. and Ozel, G. (2017) A New Exponentiated Extended Family of Distributions with Applications. Journal of Science, 30, 101-115.

[26] Hassan, A.S., Hemeda, S.E., Maiti, S.S. and Pramanik, S. (2017) The Generalized Additive Weibull-G Family of Distributions. International Journal of Statistics and Probability, 6, 65. https://doi.org/10.5539/ijsp.v6n5p65

[27] Hassan, A.S., Elgarhy, M., Nassr, S.G., Ahmad, Z. and Alrajhi, S. (2019) Truncated Weibull Fréchet Distribution: Statistical Inference and Applications. Journal of Computational and Theoretical Nanoscience, 16, 52-60. https://doi.org/10.1166/jctn.2019.7734

[28] Abid, S. and Abdulrazak, R. (2017) Truncated Fréchet-G Generator of Distributions. Applied Mathematics, 7, 51-66.

[29] Mansoor, M., Tahir, M.H., Cordeiro, G.M., Alzaatreh, A. and Zubair, M. (2017) A New Family of Distributions to Analyze Lifetime Data. Journal of Statistical Theory and Applications, 16, 490-507. https://doi.org/10.2991/jsta.2017.16.4.6

[30] Haq, M.A. and Elgarhy, M. (2018) The Odd Fréchet-G Family of Probability Distributions. Journal of Statistics Applications \& Probability, 7, 185-201. https://doi.org/10.18576/jsap/070117

[31] Hassan, A.S. and Nassr, S.G. (2019) Power Lindley-G Family of Distributions. Annals of Data Science, 6, 189-210. https://doi.org/10.1007/s40745-018-0159-y

[32] Zografos, K. and Balakrishnan, N. (2009) On Families of Beta and Generalized Gamma-Generated Distributions and Associated Inference. Statistical Methodology, 6, 344-362. https://doi.org/10.1016/j.stamet.2008.12.003

[33] Ristic, M.M. and Balakrishnan, N. (2012) The Gamma-Exponentiated Exponential Distribution. Journal of Statistical Computation and Simulation, 82, 1191-1206. https://doi.org/10.1080/00949655.2011.574633

[34] Saboor, A., Bakouch, H.S. and Khan, M.N. (2016) Beta Sarhan-Zaindin Modified Weibull Distribution. Applied Mathematical Modelling, 40, 6604-6621. https://doi.org/10.1016/j.apm.2016.01.033

[35] Alizadeh, M., Merovci, F. and Hamedani, G.G. (2017) Generalized Transmuted Family of Distributions: Properties and Applications. Hacettepe Journal of Mathematics and Statistics, 46, 645-667. https://doi.org/10.15672/HJMS.201610915478 
[36] Alizadeh, M., Cordeiro, G.M., Nascimento, A.D.C., Lima, M.C.S. and Ortega, E.M.M. (2017) Odd-Burr Generalized Family of Distributions with Some Applications. Journal of Statistical Computation and Simulation, 87, 367-389. https://doi.org/10.1080/00949655.2016.1209200

[37] Jamal, F., Nasir, M.A., Tahir, M.H. and Montazeri, N.H. (2017) The Odd Burr-III Family of Distributions. Journal of Statistics Applications \& Probability, 6, 105-122. https://doi.org/10.18576/jsap/060109

[38] Bakouch, H.S., Chesneau, C. and Khan, M.N. (2018) The Extended Odd Family of Probability Distributions with Practice to a Submodel. Filomat, 33, 3855-3867. https://doi.org/10.2298/FIL1912855B

[39] Zaninetti, L. and Ferraro, M. (2008) On the Truncated Pareto Distribution with Application. Central European Journal of Physics, 6, 1-6.

[40] Zhang, T. and Xie, M. (2011) On the Upper Truncated Weibull Distribution and Its Reliability Implications. Reliability Engineering and System Safety, 96, 194-200. https://doi.org/10.1016/j.ress.2010.09.004

[41] Burroughs, S.M. and Tebbens, S.F. (2002) The Upper-Truncated Power Law Applied to Earthquake Cumulative Frequency-Magnitude Distributions. Bulletin of the Seismological Society of America, 92, 2983-2993.

https://doi.org/10.1785/0120010191

[42] Aban, I.B., Meerschaert, M.M. and Panorska, A.K. (2006) Parameter Estimation for the Truncated Pareto Distribution. Journal of the American Statistical Association, 101, 270-277. https://doi.org/10.1198/016214505000000411

[43] Aldahlan, M.A. (2019) Type II Truncated Fréchet Generated Family of Distributions. International Journal of Applied Mathematics, 7, 221-228. 